\title{
The wide field imaging with VISTA and the ESO Public Surveys
}

\author{
Magda Arnaboldi ${ }^{1}$ \\ European Southern Observatory (ESO) \\ Karl Schwarzschild Str. 2, 85748 Garching Germany \\ E-mail: marnaboleeso.org
}

Observational astronomy is witnessing an era of massive surveys: SDSS, UKIDSS, PanSTARRS, SkyMapper, and LSST to only name a few of the major projects which are all investments in survey systems that range from dedicated telescopes and instruments to data processing and distribution. The common goal to all these projects is to target new science in a large variety of fields and serving broad communities. VISTA and VST public surveys are ESO's response to these new demands. In the scheme of ESO service mode operations the public surveys represent a challenge because they require the definition of several thousands of observing blocks that need to be managed, scheduled and executed in the most efficient way.

In this paper we present the ESO survey telescopes VISTA and VST, the ESO public survey projects, their areal coverage and survey depths, and their coordination with the survey projects world wide. We then outline the new Phase 2 tools being developed to support the service mode operations for public surveys, and the starting of the survey operations with VISTA. We then conclude with the presentation of the Phase 3 as an important new step towards the publication of survey data products and closing the loop with the Astronomical community at large.

ISKAF2010 Science Meeting - ISKAF2010

Assen, the Netherlands

June 10-14 2010

Speaker 


\section{Introduction}

In this proceeding, we shall present the ESO telescope facilities VISTA (4 m telescope, near infrared) and VST (2.6 m telescope, optical) that are devoted to the data acquisition in service mode for to public surveys, as more than $75 \%$ of the ESO time on these telescopes will be allocated to these collaborative projects. We shall then present the nine ESO public surveys, their areal coverage and survey depths, and a brief summary of the survey projects currently planned or started worldwide. The ESO public survey projects within Europe target many of the fundamental questions in astrophysics today, ranging from the nature of dark energy to the universality of the stellar initial mass function.

One important aspect of any public surveys is the legacy value and the availability/easy access of the public surveys' data products by the whole Astronomical community. Because of the very large volume, the access to the raw data is only the first step towards the scientific use of these data. To increase the scientific use of the public surveys' data products ESO is developing policies and infrastructures that go under the name of Phase 3. The Phase 3 allows the Public Survey teams to submit, validate and publish data products from surveys via the ESO Science Archive Facility, and facilitates their retrival by the Astronomical community via a dedicated query interface.

\section{The ESO survey telescope facilities: VST and VISTA}

\subsection{VLT Survey Telescope - VST}

The VLT Survey Telescope (VST) is a 2.6 metre optical telescope that will be fitted with a large camera (OmegaCAM) comprised of 32 separate CCD chips (280 Megapixels). This enormous camera has a field-of-view of 1 square degree with a pixel scale of 0 ".2 arcseconds. The large field-of-view is ideally suited for surveying large areas. The camera will be equipped with 5 broadband filters ( $\left.u^{\prime}, g^{\prime}, r^{\prime}, i^{\prime}, z^{\prime}\right)$ as well as an $\mathrm{H} \alpha$ filter, a Strömgren $v$ filter, Johnson B and V filters, and a 4-segmented u'g'r'i' filter for photometric monitoring. More information of the VST and OmegaCAM are provided by the following papers on the ESO Messenger: Capaccioli et al. (2005, Messenger, 120, 10) and Cappellaro (2005, Messenger, 120, 13). The current timeline for VST foresees the start of the telescope commissioning at the end of 2010.

\subsection{Visible and Infrared Survey Telescope for Astronomy - VISTA}

The Visible and Infrared Survey Telescope for Astronomy (VISTA) is a 4 meter near-infrared (NIR) optimized telescope that is equipped with a large array of 16 infrared detectors that will fill a 1.5 square degree field (after stepping to fill in the gaps between the detectors). The VISTA filter set includes $\mathrm{Z}, \mathrm{Y}, \mathrm{J}, \mathrm{H}, \mathrm{K}_{\mathrm{s}}$ and a narrow band filter at 1.18 microns. Each exposure will contain 67 Megapixels, and a typical observing night will see the collection of about 300 GB of data. This enormous data volume requires very efficient processing and considerable resources. A good overview of the whole VISTA project can be found in Emerson, J., McPherson, A., Sutherland, W. (2006, ESO Messenger, 126, 41).

The science commissioning of VISTA started on Oct. 1, 2009, and the science verification, consisting of two dedicated mini surveys was executed from Oct. 15 to Nov. 3, 2009. Dry runs soon followed starting from Nov. 4, 2009, and the first Phase 2 call for VISTA service mode observations was issued on Dec. 22, 2009. VISTA science operations officially started on Apr. 
1, 2010 and the observations of the VISTA public surveys are now carried out routinely every night.

\section{Survey projects at ESO and worldwide}

\subsection{The VISTA surveys}

The initial phase of VISTA operation consists of the execution of six public surveys covering a range of Galactic and extra-galactic science goals, and expectations are for them to be completed in 5 years. The distribution of the survey field locations is shown in Figure 1. The areal coverage and approximate survey depths are given in Table 1.

Table 1: Parameters of the six VISTA public surveys.

\begin{tabular}{|c|c|c|c|c|c|c|}
\hline \multirow{2}{*}{ Survey } & \multirow{2}{*}{ Area $\left(\mathrm{deg}^{2}\right)$} & \multicolumn{5}{|c|}{ Filters and 5- $\sigma$ point source limits } \\
\cline { 3 - 7 } & & $\mathrm{Z}$ & $\mathrm{Y}$ & $\mathrm{J}$ & $\mathrm{H}$ & $\mathrm{Ks}$ \\
\hline $\begin{array}{c}\text { Ultra- } \\
\text { VISTA }\end{array}$ & 0.73 & & 26.7 & 26.6 & 26.1 & 25.6 \\
\hline VHS & 20000 & & 21.2 & 21.1 & 20.6 & 20.0 \\
\hline VIDEO & 12 & 25.7 & 24.6 & 24.5 & 24.0 & 23.5 \\
\hline VVV & 520 & 22.5 & 21.9 & 21.1 & 19.6 & 20.0 \\
\hline VIKING & 1500 & 23.1 & 22.3 & 22.1 & 21.5 & 21.2 \\
\hline VMC & 184 & & 23.3 & 23.0 & & 22.9 \\
\hline
\end{tabular}

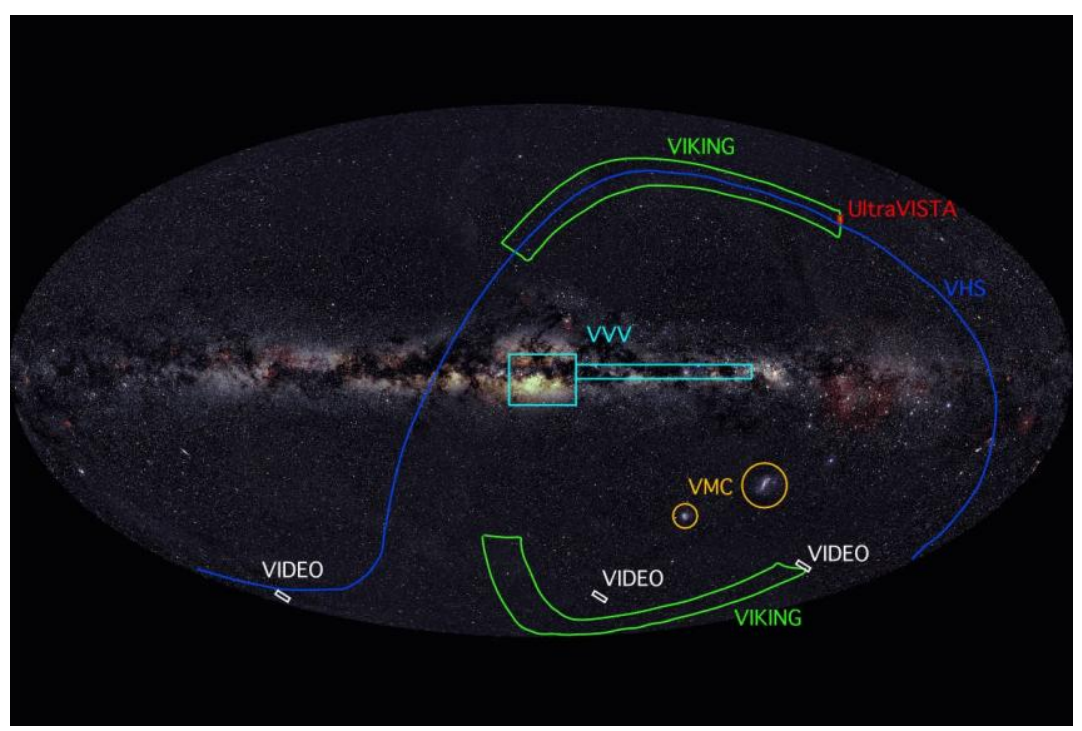

Figure 1: The sky distribution of the VISTA public surveys (VHS covers the whole Southern sky).

\subsubsection{Ultra-VISTA}

Ultra-VISTA targets a single VISTA pointing to the limits of the deepest photometry accessible to VISTA. The deepest part of the survey will only cover half the field by column-stepping, with a shallower side-ways step to fill the area and provide photometric cross-checks. The aim is to study the early Universe between redshifts 6 and 10, and a complementary narrow-band filter will search for Lyman-alpha emission at a redshift of $8.7(1.18 \mu \mathrm{m}$ filter $)$. The total number of nights requested in 5 years is about 200. 


\subsubsection{VISTA Hemisphere Survey (VHS)}

The VISTA Hemisphere Survey aims to provide a complete census of the southern sky to Ks $<$ 20, comparable to the UKIDSS LAS. The primary scientific goals of this survey are to catalogue the distribution of low mass stars, and to search for rare bright objects at high redshifts $(z>7)$. The survey will also form a NIR complement to the Dark Energy Survey (DES), allowing good photometric redshifts to be determined for large numbers of galaxies at $\mathrm{z}<1$ with a view to studying the Dark Energy equation of state. The total number of nights requested over 5 year is about 340 .

\subsubsection{VISTA Deep Extragalactic Observations (VIDEO)}

VIDEO will provide deep photometry for three strategically chosen deep fields (the XMMLSS, ELAIS-S1 and CDFS-S) for which complementary multi-wavelength data is available from X-ray to radio wavelengths. The goals are to study the details of the evolution of galaxies, large-scale structure, and AGN at high redshifts. The total numer of nights planned for the survey completion is about 160 nights.

\subsubsection{VISTA Variables in the Via Lactea (VVV)}

VVV will survey 520 square degrees of the Galactic plane over multiple epochs to search for high-proper motion stars and create a 3-dimensional map of the Galactic bulge using distance measurements to RR-Lyrae stars. The survey will provide age-dating of the various stellar populations in the Galactic bulge, and an evolutionary perspective on Galactic clusters. The number of nights required for the completion of the survey is about 210.

\subsubsection{VISTA Kilo-degree Infrared Galaxy survey (VIKING)}

VIKING complements the proposed VST KIDS survey, providing NIR photometry to moderate depths over two large contiguous fields at high Galactic latitude. This survey will be used to study galaxy evolution, and may form the basis for a spectroscopic survey to study the phenomenon of Baryonic Acoustic Oscillations (BAOs) to provide a geometric constraint of Dark Energy. The number of nights required for the execution of the survey is about 240 distributed over 5 year.

\subsubsection{VISTA survey of the Magellanic Cloud system (VMC)}

This survey will cover the Magellanic Clouds, the bridge between them, and the diffuse Magellanic Stream with 15 different epochs to study the evolution of the stellar populations and obtain insights into the history of the interactions between the Clouds and between the Clouds and the Milky Way. The number of nights requested for the excution of the VMC survery is about 200 nights over 5 years.

\subsection{The VST Surveys}

There are three VST Public surveys approved by the ESO Director General, and they are described below. In Figure 2 we show the sky coverage of the three VST public surveys, overlaid on a 2MASS image of the southern sky. It is anticipated that the surveys will take approximately 3 years to be completed and their areal coverage and survey depths are summarized in Table 2. 


\subsubsection{The Kilo-Degree Survey (KIDS)}

This survey aims to image 1500 square degrees in 4 optical bands, to be complemented in the near-infrared with data from the VISTA VIKING survey. The survey depth is 2.5 magnitudes deeper than the Sloan Digital Sky Survey (SDSS), with considerably better image quality. The primary science driver for the design of this project has been weak gravitational lensing. The science goals of the KIDS project are numerous, including studying dark matter halos and dark energy with weak lensing, investigating galaxy evolution, searching for galaxy clusters, and looking for high redshift quasars. The KIDS project fills an important niche in lensing surveys between smaller, slightly deeper surveys, such as the CFHT Legacy Survey, and larger, shallower surveys like the SDSS.

\subsubsection{The VST ATLAS}

This survey is targeting 4500 square degrees of the Southern Sky in 5 filters to depths comparable to the SDSS. It will be complemented with near-infrared data from the VHS VISTA survey. The primary science driver is to determine the dark energy equation of state by examining the 'baryon wiggles' in the matter power spectrum, via surveys of luminous red galaxies using both photometric and spectroscopic redshifts. This survey will also provide the imaging base for many other future spectroscopic surveys, both at the VLT and also via widefield fibre spectrographs such as the AAOmega instrument at the Anglo-Australian Observatory.

\subsubsection{The VST Photometric H- $\alpha$ Survey of the Southern Galactic Plan (VPHAS+ )}

This survey will combine $\mathrm{H} \alpha$ and broadband u'g'r'i' imaging over an area of 1800 square degrees capturing the whole of the Southern Galactic Plane within the latitude range $|b|<5$ degrees. VPHAS+ will facilitate detailed extinction mapping of the Galactic Plane, and can be used to map the structure of the Galactic disk and its star formation history. The survey will yield a catalogue of around 500 million objects, which will include greatly enhanced samples of rare evolved massive stars, Be stars, Herbig and T Tau stars, post-AGB stars, compact nebulae, white dwarfs and interacting binaries. This survey is complementary to IPHAS, a survey of the Northern Galactic Plane nearing completion, but VPHAS+ will include more filters and will achieve better image quality.

Table 2: Parameters of the three VST public surveys.

\begin{tabular}{|c|c|c|c|c|c|c|c|}
\hline \multirow[t]{2}{*}{ Survey } & \multirow[t]{2}{*}{ Area $\left(\mathrm{deg}^{2}\right)$} & & \multicolumn{5}{|c|}{$\begin{array}{c}\text { Filters and } 10-\sigma \text { point source } \\
\text { limits } \\
\text { (AB magnitudes })\end{array}$} \\
\hline & & $\mathrm{u}^{\prime}$ & $g^{\prime}$ & r' & i' & $\mathrm{H} \alpha$ & $\mathrm{z}^{\prime}$ \\
\hline KIDS & 1500 & 24.1 & 24.6 & 24.4 & 23.4 & & \\
\hline $\begin{array}{c}\text { VST } \\
\text { ATLAS }\end{array}$ & 4500 & 22.0 & 22.2 & 22.2 & 21.3 & & 20.5 \\
\hline VPHAS+ & 1800 & 21.8 & 22.5 & 22.5 & 21.8 & 21.6 & \\
\hline
\end{tabular}




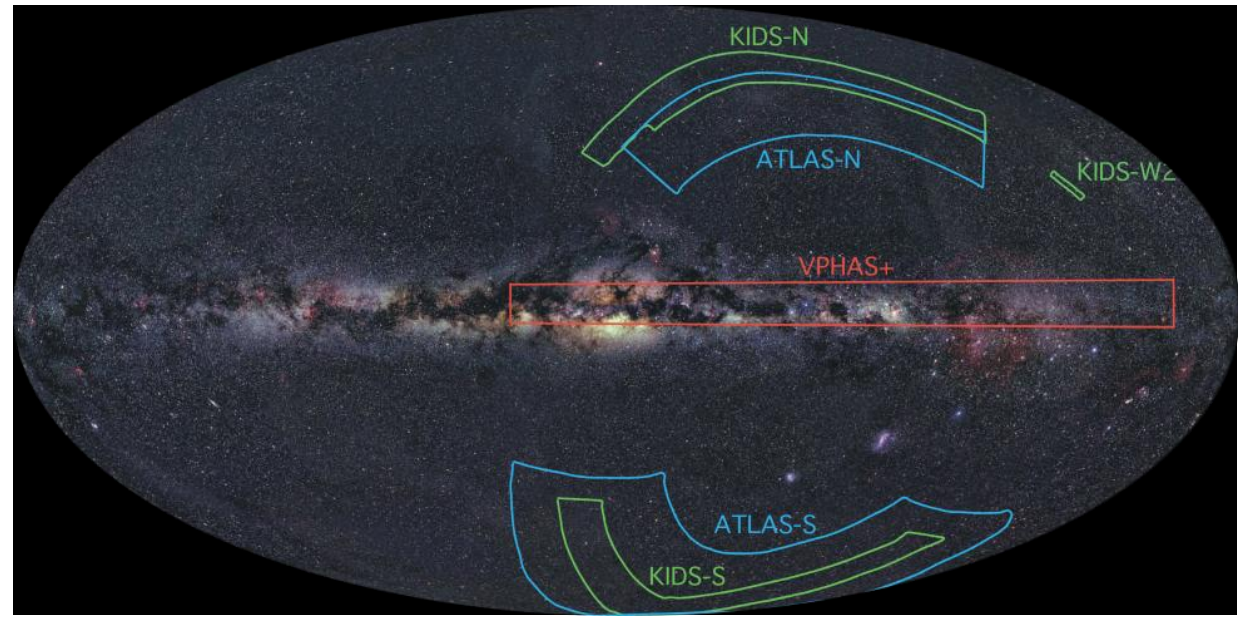

Figure 2 - The sky coverage of the three VST PS, overlaid on a 2MASS image of the southern hemisphere

\subsection{Survey projects worldwide}

The six VISTA plus three VST surveys in the southern hemisphere are the European projects that address a demand coming from the Astronomical community and involve more than 300 astronomers as PIs and Co-Is. Such demands at the international level lead to the definition and implementation of telescope facilities and several survey projects in addition to those presented thus far. Among these projects we list here the Dark Energy Survey (DES) project at the $4 \mathrm{~m}$ CTIO telescope, that have a strong synergy with VHS (5000 sq deg), the Pan-SStar1 survey aiming at covering the whole northern sky in the visible bands, and the Stromlo Southern Sky Survey, with similar goals as Pan-SStar1 for the southern sky. In Table 3 we provide a summary of the telescope facilities for surveys worldwide and the telescope diameter, field-of-view (FoV), Entendu, pixel size and wavelength range values for comparison with VISTA and VST.

\section{Phase 2 for ESO public surveys}

The ESO public surveys on VISTA and VST are ambitious projects which range from surveys over a very wide area covered with short exposures, like the VISTA Hemisphere Survey (VHS) survey that aims at covering the whole southern hemisphere, to deep surveys concentrating to small areas but going very deep. Typical examples of the latter are the Ultra-VISTA and VISTA Deep Extragalactic Observations (VIDEO) surveys. Both wide and deep surveys are similar in terms of the amount of total observing time, although their observing requirements, i.e. seeing, sky transparency, moon illumination and RA range may be complementary. The size of survey projects has a strong influence on their operations. To put things in perspective the widest area survey VHS wishes to cover 17,700 sq. degrees which according to their observing strategy translates into $\sim 3200$ hours. The survey with the largest expected number of OBs is VVV (VISTA Variables in the Via Lactea) which aims at covering 520 sq. degrees in the Galactic bulge and disk, over 80 epochs and $\sim 1930$ hrs total observing time which translates into more than 30,000 OBs (the unit observation at ESO telescopes is called Observing Block - OB) The deepest survey, Ultra-VISTA, while insisting on a single pointing on the sky $(0.73 \mathrm{sq}$. degrees) requires about $\sim 1800 \mathrm{hrs}$ of observing time total to reach very faint limiting fluxes, i.e. $25.6 \mathrm{mag}_{\mathrm{AB}}(\mathrm{Ks})$ and $>26 \mathrm{mag}_{\mathrm{AB}}(\mathrm{YJH})$. When compared with the average numbers of scheduled OBs, about 3000-5000 OBs per year per UT in the standard VLT 
service mode operations, the impact of the ESO public survey on the Paranal science operations becomes very clear.

Table 3: telescope facilities for surveys worldwide and their characteristics.

\begin{tabular}{|c|c|c|c|c|c|}
\hline & $\begin{array}{l}\text { Telescope } \\
\text { Diameter }\end{array}$ & FoV & $\begin{array}{l}\text { Etendu } \\
{\left[\mathrm{m}^{2} \mathrm{deg}^{2}\right]}\end{array}$ & $\begin{array}{l}\text { Pixel size } \\
\text { [arcsec/pixel] }\end{array}$ & $\begin{array}{l}\text { Wavelength } \\
\text { range }[\mu \mathrm{m}]\end{array}$ \\
\hline SkyMapper & 1.4 & $5.7 \mathrm{deg}^{2}$ & 6.6 & 0.500 & $0.32-0.95$ \\
\hline Pan-STARRS 1 & 1.8 & $2.8^{\circ} \times 2.9^{\circ}$ & 16.3 & 0.300 & $0.4-1.15$ \\
\hline SDSS & 2.5 & & 6.0 & 0.394 & $0.33-1.0$ \\
\hline MegaCam & 3.6 & $0.96^{\circ} \times 0.94^{\circ}$ & 7.6 & 0.187 & $0.34-0.95$ \\
\hline CTIO (DES) & 4.0 & $4.0 \mathrm{deg}^{2}$ & 16 & $\sim 0.20$ & $0.6-1.0$ \\
\hline VISTA & 4.0 & $\begin{array}{l}1.65^{\circ} \mathrm{deg} \\
\text { circ }\end{array}$ & 5.2 & 0.339 & $0.88-2.15$ \\
\hline SuprimeCam & 8.0 & $34^{\prime} \times 27^{\prime}$ & 13.5 & 0.200 & $0.36-1$ \\
\hline VLT HAWK-I & 8.2 & $7.5^{\circ} \times 7.5^{\prime}$ & 0.81 & 0.106 & $1-2.2$ \\
\hline VLT-VIMOS & 8.2 & $4 \times 7^{\circ} \times 8^{\prime}$ & 3.2 & 0.205 & $0.36-1$ \\
\hline$L B C @ L B T$ & 8.4 & $23^{\prime} \times 23^{\prime}$ & 8.1 & 0.230 & $0.32-1.0$ \\
\hline$V S T$ & 2.6 & $1 \mathrm{deg}^{2}$ & 5.5 & 0.210 & $0.33-1.0$ \\
\hline $\begin{array}{l}\text { Subaru } \\
\text { HyperSuprime }\end{array}$ & 8.0 & $1.5 \mathrm{deg}$ circ & 94 & 0.17 & $0.36-1$ \\
\hline LSST & 8.4 & $3.5 \mathrm{deg}$ circ & 319 & 0.200 & $0.33-1.07$ \\
\hline
\end{tabular}

\subsection{The Phase 2 tools}

The generation of thousands of OBs can be simplified when the geometry definition of a survey area , i.e. where to point on the sky, is separated from the definition of the observing strategy, i.e. filter selection, epochs, order of execution, seeing and trasparency requirements. In order to support service mode observations for public surveys, ESO and the VISTA consortium developed the new capabilities within the ESO P2PP (Phase 2 Proposal Preparation tool) and the SADT - Survey Area Definition tool. These tools are currently deployed for VISTA operations, and they will be upgraded to support the VST operations also.

For the preparation of the observations for Public Surveys, users define the geometry of the survey areas using SADT. The output of SADT is then imported in the new P2PP for Surveys and used to prepare valid OBs.

\subsubsection{The Survey Area Definition Tool (SADT)}

The Survey Area Definition Tool - SADT for short - is an utility developed by the VISTA consortium that allows users to define areas for surveys to be executed with either VIRCAM at 
VISTA or OmegaCam at the VST, according to a number of criteria. The SADT determines the central coordinates of the different pointings required to cover the field with the constraints set by the user, as well as ancillary guide star information for the field acquisition and telescope guiding. The output from SADT is a file to be ingested by P2PP, such file contains all the target information required for the preparation of the OBs.

The IR detectors in VISTA's focal plane are not contiguous, see Figure 3 for an overview of the detectors' geomery in the VISTA focal plane. Therefore a single sky exposure shows large gaps between the detectors - it is known as a 'pawprint'. To make an image without gaps it is necessary to combine several (minimum six) pawprints with position offsets so as to cover the gaps and form a filled 'tile', see Figure 3 for a graphycal illustration of VISTA pawprints and tiles. To cover a survey area, the positions of the tiles must be computed and suitable candidate guide stars and active optics $(\mathrm{aO})$ stars must be pre-selected for each pawprint in a given tile. The guide stars and $\mathrm{AO}$ stars are then ready for input when the observation is carried out at the telescope, which ensures that the survey execution is not slowed down by frequent operator intervention.
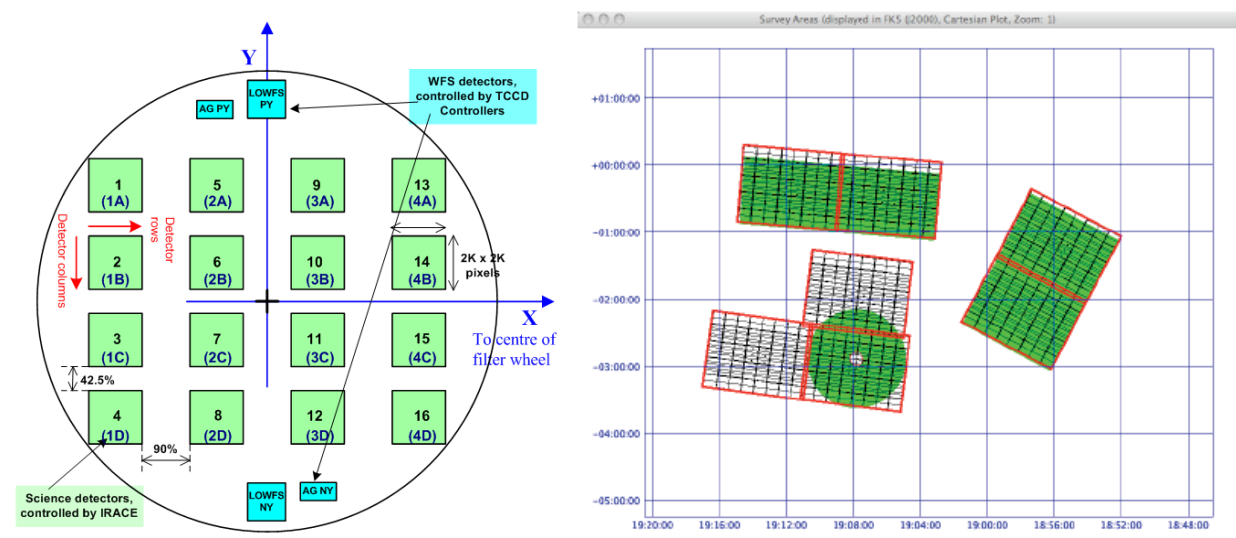

Fig. 3: Left: Overview of geometry of the VIRCAM detectors, autoguiding and wavefront sensors in the VISTA focal plane. Left: Pawprints (black contours) and tiles (red squares) computed for the survey area indicated by the full green regions.

\subsubsection{P2PP for surveys}

For the preparation of the VISTA and VST surveys OBs, the survey definition generated by SADT is given as input to P2PP. Then P2PP combines the survey definition with parent OBs, i.e. OBs whose exposure times, filters, dithering patterns and observing constraints are set, but lack pointing information. The parent OBs can be a single OB, or more OBs, which are then structured in an observing strategy. The observing strategy may require grouping of OBs with different priorities, chains of OBs, or a time sequence of OBs. The new version of P2PP allows the implementation of these different observing strategies via the scheduling containers defined as time-links, concatenations and groups of OBs. Time-link allows to definition of a sequence of OBs, according either to a relative or an absolute time sequence. A concatenantion of OBs is a set of OBs that must be executed back-to-back, without any break and specific order of execution. A group of OBs defines a set of OBs according to a joint score that allows the completion of the OBs within such group before any other OBs from the same program are executed.

During the first official Phase 2 for survey (P84+) 4280 ${ }^{\mathrm{i}}$ OBs were submitted and reviewed. 


\section{Public survey data flow and the Phase 3 for ESO public surveys}

\subsection{VISTA data flow}

Once the VISTA OBs are executed at the Paranal Observatory in Chile, the raw data are shipped to the ESO headquarters in Garching, Germany, where they are copied in the ESO archive, and then are shipped to Cambridge for the full science data reduction. Once the raw data are in ESO Garching, they are further checked by the Quality Control pipelines and the health check monitoring for the instrument trends. In Figure 4 we show a summary diagram of the monthly data flow from all the Paranal UTs and instruments compared with the raw data generated by the Survey telescopes, VISTA and VST.

Once in Cambridge, UK, the Cambridge Astronomical Survey Unit (CASU: http://www.ast.cam.ac.uk/vdfs/) implements the data processing aspects of VISTA observations' and the PIs may access the processed data after the CASU step. After the instrument-processing steps are executed, the reduced VISTA data are transferred to Edinburgh where the Wide Field Astronomy Unit (WFAU: http://www.roe.ac.uk/ nch/wfcam/) is responsible for the design and implementation of the survey science-oriented data reduction.

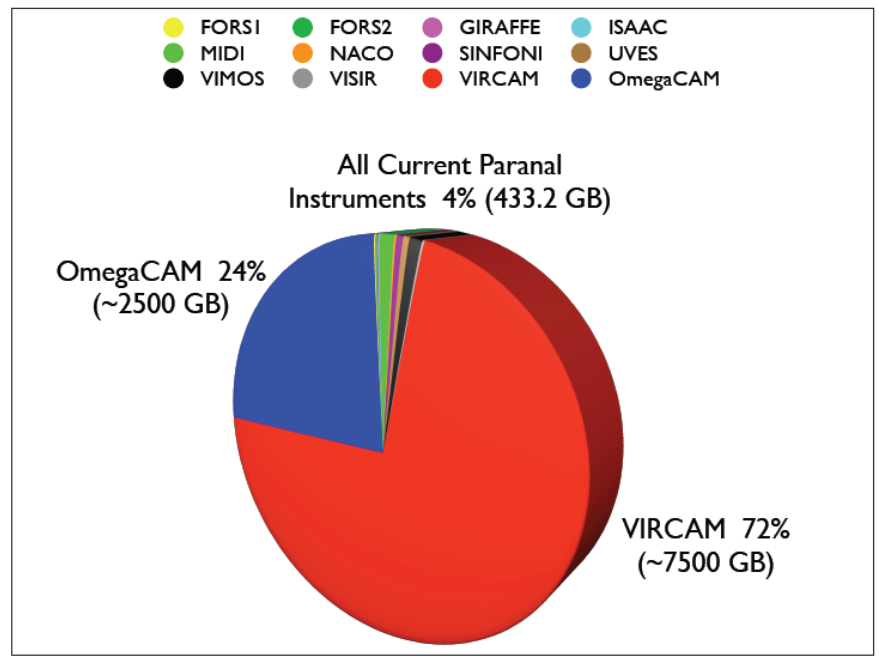

Fig. 4: Monthly dataflow from Paranal UTs and survey telescopes; from Arnaboldi et el. (2007, Messenger 127, 28)

\subsection{Phase 3}

The Phase 3 represents the final step in the execution of ESO Large Programs and Public Surveys, it is a joint effort of the PIs/CoIs and ESO and closes the loop with the Astronomical community. The ESO commitment to the success of the public surveys does include the development of the Phase 3 infrastructure. This infrastructure combines guidelines that set the relevant standards for the format of data products and metadata definitions and the deployment of tools for validation and delivery of data products. The Phase 3 includes the availability of dedicated query interfaces in the ESO archive for the retrieval of catalogs and surveys data products to ease community access to the public survey science data.

In addition to the fast availability of the raw data that becomes public as soon as they are ingested into the ESO archive the Phase 3 deliveries from the ESO Public Surveys consist of the science data products and the quality control reports described and agreed upon in the Survey Management Plans. The timeline for data products delivery is described in the contracts signed 
by ESO Director General and Public Survey PIs. These milestones for the data delivery are associated with and in preparation to the survey progress reviews by the Public Survey Panel and the ESO Observation Program Committee (OPC) for approval of further time allocation at the survey telescopes.

In the specific, science data products from public surveys consist of:

- Astrometrically and photometrically calibrated, co-added, re-gridded images, along with their respective weight maps, in all of the project-relevant filters.

- Aperture-matched source catalogues based on individual or co-added bands. Associated source catalogues linking the parameters of individual objects across all of the observed filter bands.

- Light curves for multi-epoch observations of variable sources.

\section{Conclusion}

ESO's support to public surveys foresees an end-to-end system which starts from the submission of the survey's letter of intent by interested teams, the approval of the survey proposals and survey management plans, to a robust service mode observation/data acquisition at the telescope, and then finally with the publication of the public survey data products through the ESO Science Archive Facility. This coordinated support is now in place for imaging surveys with VISTA and VST and will soon be adopted for the ESO spectroscopic public surveys with the current VLT instrumentation and the ESO large programs.

\section{Acknowledgements}

The author would like to thank the organisers of the ISKAF 2010 science meeting for the invitation to give the review of the ESO public survey projects. The author is indebited to Gavin Dalton, Jim Emerson, Michael Irwin, William Sutherland and the ESO Survey team (Evanthia Hatziminaoglou, Michael Hilker, Wolfgang Hummel, Gaitee Hussain, Mark Neeser, Monika Petr-Gotzens, Marina Rejkuba), the Paranal Science Operation - VISTA telescope (Valentin Ivanov, Steffen Mieske ,Thomas Szeifert), the ESO External Data Group (Joerg Retzlaff, Remco Slijkhuis), Fernando Comeron, Martino Romaniello and Thomas Bierwirth.

\section{References}

[1] Arnaboldi, M., et al. 2007, ESO Messenger, 127, 28

[2] Capaccioli, M., Mancini, D., Sedmak, G 2005, ESO Messenger, 120, 10

[3] Cappellaro, E. 2005, Messenger, 120, 13

[4] Emerson, J., McPherson, A., Sutherland, W. 2006, ESO Messenger, 126, 41

${ }^{i}$ Number of OBs equivalent to all service mode OBs for one UT in one year! 